\title{
A host-vector system for analysis and manipulation of rifamycin polyketide biosynthesis in Amycolatopsis mediterranei
}

\author{
Zhihao Hu, ${ }^{1}$ Daniel Hunziker, ${ }^{1}$ C. Richard Hutchinson ${ }^{3,4}$ \\ and Chaitan Khosla ${ }^{1,2}$
}

1,2 Departments of Chemical Engineering ${ }^{1}$, Chemistry and Biochemistry ${ }^{2}$, Stanford University, Stanford, CA 94305-5025, USA

3,4 School of Pharmacy ${ }^{3}$ and Department of Bacteriology 4 , University of Wisconsin, Madison, WI 53706, USA
Author for correspondence: Chaitan Khosla. Tel: +1 6507236538 . Fax: +16507236538. e-mail: ck@chemeng.stanford.edu

Modular polyketide synthases (PKSs) are a large family of multifunctional enzymes responsible for the biosynthesis of numerous bacterial natural products such as erythromycin and rifamycin. Advanced genetic analysis of these remarkable systems is often seriously hampered by the large size ( $>40 \mathrm{~kb}$ ) of PKS gene clusters, and, notwithstanding their considerable fundamental and biotechnological significance, by the lack of suitable methods for engineering non-selectable modifications in chromosomally encoded PKS genes. The development of a facile host-vector strategy for genetic engineering of the rifamycin PKS in the producing organism, Amycolatopsis mediterranei S699, is described here. The genes encoding all 10 modules of the rifamycin PKS were replaced with a hygromycin-resistance marker gene. In a similar construction, only the first six modules of the PKS were replaced. The deletion hosts retained the ability to synthesize the primer unit 3-amino-5-hydroxybenzoic acid (AHBA), as judged by co-synthesis experiments with a mutant strain lacking AHBA synthase activity. Suicide plasmids carrying a short fragment from the 5' flanking end of the engineered deletion, an apramycin-resistance marker gene, and suitably engineered PKS genes could be introduced via electroporation into the deletion hosts, resulting in the integration of PKS genes and biosynthesis of a reporter polyketide in quantities comparable to those produced by the wild-type organism. Since this strategy for engineering recombinant PKSs in $A$. mediterranei requires only a selectable single crossover and eliminates the need for tedious non-selectable double-crossover experiments, it makes rifamycin PKS an attractive target for extensive genetic manipulation.

Keywords: rifamycin, polyketide synthase, Amycolatopsis

\section{INTRODUCTION}

Polyketides are a large family of microbial natural products especially abundant in actinomycetes. Medicinally important polyketides produced by species belonging to the genus Amycolatopsis include rifamycin (Sensi et al., 1959), vancomycin (Barna \& Williams, 1984), balhimycin (Nadkarni et al., 1994) and dethymicin (Ueno et al., 1992). Among these, rifamycin and its derivatives are of particular interest, as they can be used to treat major infectious diseases such as tuberculosis

Abbreviations: AHBA, 3-amino-5-hydroxybenzoic acid; PKS(s), polyketide synthase(s). and leprosy (Cole, 1996). They are also becoming increasingly important in the control of infections occurring in organ implant and AIDS patients. The glycopeptide antibiotics vancomycin and balhimycin have antibacterial activity against methicillin-resistant Staphylococcus aureus strains, whereas dethymicin is an immunosuppressant with a mode of action different from cyclosporin, FK506, rapamycin and other such immunosuppressants.

The mechanism of action of rifamycin against bacteria and viruses (Lowder \& Johnson, 1987; Riva et al., 1972) as well as its structure-activity relationships (Arora \& Main, 1984; Arora, 1985; Bacchi et al., 1998; Bartolucci et al., 1995) have been extensively studied. More 


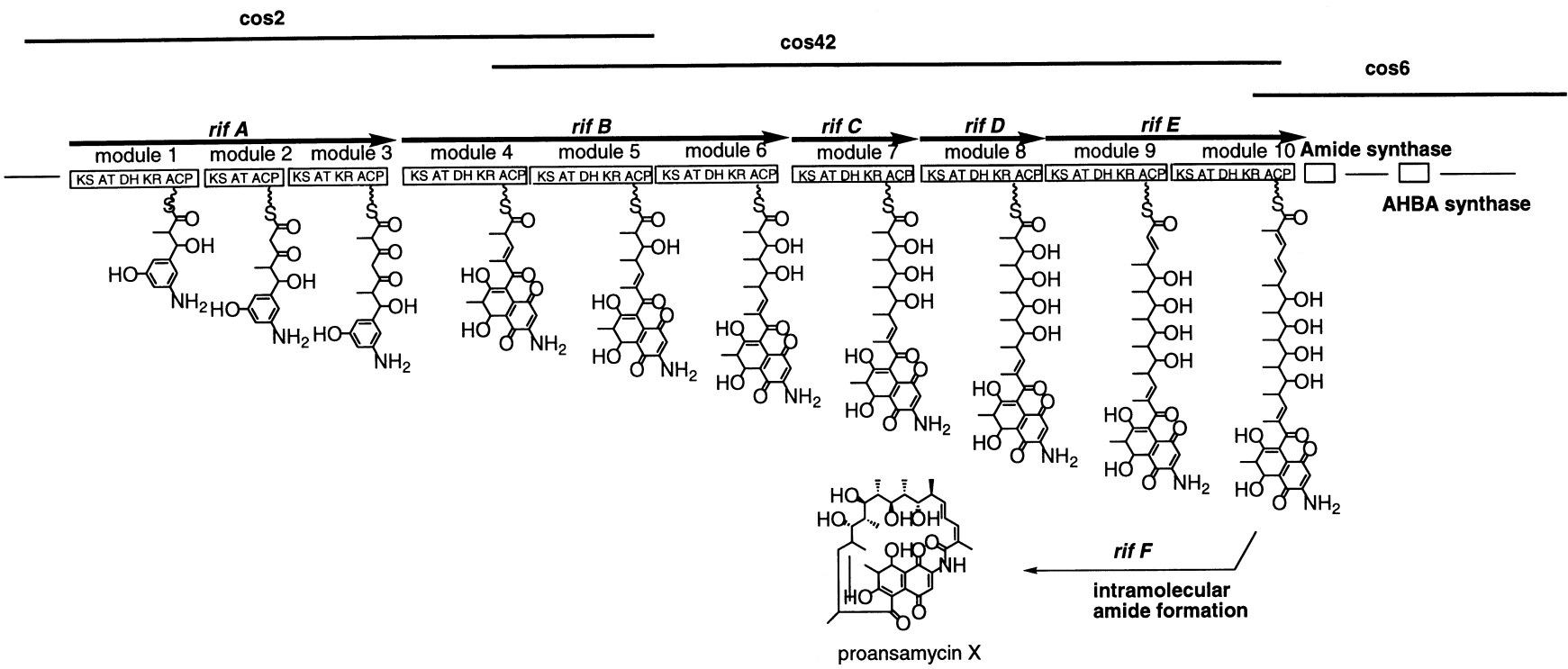

Fig. 1. Rifamycin PKS gene cluster. The three cosmids comprising the rifamycin PKS gene cluster, cloned in this study, are shown at the top. Beneath them are the genes encoding the rifamycin PKS pathway, together with their inferred biosynthetic intermediates. The product of the rifamycin PKS pathway is believed to be proansamycin X, although this remains to be directly confirmed. Recently it has been suggested that cyclization of the polyketide intermediate to generate the naphthalene functionality occurs between the tetraketide and pentaketide stages on the PKS-bound intermediate (Yu et al., 1999). KS, Ketosynthase; AT, acyltransferase; DH, dehydratase; KR, ketoreductase; ACP, acyl carrier protein.

recently, the biosynthesis of rifamycin has attracted attention. The polyketide chain starts with a 3-amino-5hydroxybenzoic acid (AHBA) primer unit (Ghisalba \& Nuesch, 1981; Ghisalba et al., 1981; Hatano et al., 1982). Initially, the gene encoding AHBA synthase, which catalyses the last step in AHBA biosynthesis, was cloned (Kim et al., 1998); in turn, this led to cloning and sequencing of the whole gene cluster (Schupp et al., 1998; Tang et al., 1998). Encoded within the gene cluster is a 10-module polyketide synthase (PKS) presumably responsible for the biosynthesis of proansamycin $\mathrm{X}$, the polyketide precursor of rifamycin B (Fig. 1). This multifunctional enzyme complex presents an attractive opportunity for engineering novel rifamycin analogues, as has been repeatedly demonstrated for the betterstudied erythromycin PKS (Cane et al., 1998). However, a central prerequisite for such genetic engineering is the availability of suitable genetic tools and methods.

Two basic strategies have been exploited for the genetic manipulation of erythromycin biosynthesis. One involves using the natural producer of erythromycin Saccharopolyspora erythraea. Chromosomal mutants in the erythromycin PKS genes are generated through standard homologous recombination-mediated gene replacement methods. The principal advantage of this approach is that the system can make fully modified and often biologically active erythromycin derivatives (Donadio et al., 1993; Ruan et al., 1997; Marsden et al., 1998). However, since homologous recombination occurs at a relatively low frequency, the method is tedious, especially where non-selectable double crossovers are required. An alternative strategy takes ad- vantage of a host-vector system that uses Streptomyces coelicolor CH999 as the host and pRM5 as the vector (McDaniel et al., 1993). The erythromycin PKS genes or mutants thereof are cloned into pRM5 derivatives and introduced via transformation into $\mathrm{CH} 999$ to produce the expected reporter polyketides (Kao et al., 1994, 1996; Bedford et al., 1996). Whereas only aglycones are generated using this approach, it offers the convenience of shuttle-plasmid-borne genes, and is therefore more amenable to rapid mutagenesis. Recent work by Leadlay and coworkers has sought to combine features of these two approaches (Rowe et al., 1998).

In Amycolatopsis, unlike Streptomyces, genetic tools and methods, such as transformation and transfection, and cloning vectors, are relatively undeveloped. Plasmids that replicate in Streptomyces cannot be maintained in Amycolatopsis mediterranei (Schupp \& Diver, 1986; Pelzer et al., 1997), although plasmids such as pIJ702 and pKC505 from Streptomyces could replicate in Amycolatopsis orientalis (Matsushima et al., 1987). A few indigenous plasmids such as pMEA100 (Moretti et al., 1985; Madon \& Hutter, 1991), pMEA300 (Vrijbloed et al., 1994) and pA387 (Lal et al., 1991) have been found in Amycolatopsis species, but have not yet been fully developed into cloning and expression vectors. Consequently, genetic manipulation of the rifamycin PKS genes in A. mediterranei is very difficult, and primarily relies on the use of suicide delivery systems.

Here we describe the construction of specific deletion mutants of A. mediterranei $\mathrm{S} 699$ as hosts for PKS gene 
expression. Engineered forms of the rifamycin PKS can be introduced into these hosts either on plasmid vectors or through single crossovers into the chromosome. We demonstrate the utility of this approach by reinserting the rifA gene into the chromosome to produce the expected tetraketide P8/1-OG.

\section{METHODS}

Bacterial strains and plasmids. The Escherichia coli strain used in this study was XL-1 Blue (Stratagene). Amycolatopsis mediterranei strains S699 and HGF003 were gifts from Heinz G. Floss (University of Washington, Seattle, USA). The former produces rifamycin $\mathrm{B}$, whereas the latter is a null mutant in which the AHBA synthase gene has been inactivated (Kim et al., 1998; Hunziker et al., 1998). SuperCos 1 was used as the cosmid vector for constructing the genomic library. Plasmid pIY15 contains a $7 \cdot 5 \mathrm{~kb}$ Pst I fragment from the rifamycin PKS gene cluster (August et al., 1998), and was used as a probe to isolate clones containing rif genes from the cosmid library. Plasmid pSET152, which confers apramycin resistance both in E. coli and in A. mediterranei, was used as a cloning vector in this study (Flett et al., 1997). (Note that although pSET152 contains the att integration site of the phage $\phi \mathrm{C} 31$, its use here was solely based on cloning convenience. By itself, this vector is incapable of integrating into the genome of $A$. mediterranei.)

Media, chemicals and growth conditions. E. coli strains were grown in Luria-Bertani (LB) medium at $37^{\circ} \mathrm{C}$. When necessary, $50 \mu \mathrm{g}$ carbenicillin $\mathrm{ml}^{-1}$ or $60 \mu \mathrm{g}$ apramycin $\mathrm{ml}^{-1}$ was present in the medium. A. mediterranei strains were grown in YMG (also called YM) (Lal et al., 1998) medium at $30^{\circ} \mathrm{C}$, supplemented with $60 \mu \mathrm{g}$ apramycin $\mathrm{ml}^{-1}$ and/or $50 \mu \mathrm{g}$ hygromycin $\mathrm{ml}^{-1}$ when necessary. All antibiotics were purchased from Sigma; 3-hydroxybenzoic acid was obtained from Aldrich, whereas 3-amino-5-hydroxybenzoic acid (AHBA) was synthesized in this laboratory and verified by NMR.

DNA manipulation. DNA fragments for subcloning were purified with Qiaex I (for fragments smaller than $10 \mathrm{~kb}$ ) or Qiaex II (for fragments larger than $10 \mathrm{~kb}$ ) gel extraction kits (Qiagen). DNA blotting and Southern hybridization made use of protocols recommended for the DIG labelling and detection kit (Boehringer). All other DNA manipulations utilized standard methods (Sambrook et al., 1989).

Construction of a genomic DNA library. Genomic DNA was isolated from A. mediterranei $\mathrm{S} 699$ and partially digested with Sau3A. The fraction containing $35-42 \mathrm{~kb}$ fragments was purified from a $0.3 \%$ agarose gel and ligated with BamHI/ $\mathrm{XbaI}$-linearized SuperCos 1 (Stratagene). The ligation mixture was packaged using Gigapack III Gold packaging extract (Stratagene), and used to infect E. coli XL-1 Blue. Recombinants were selected on LB agar plates containing carbenicillin.

Purification of P8/1-OG. Spores of A. mediterranei were plated on Petri dishes containing $40 \mathrm{ml}$ YMG medium to give lawns. Following growth at $30^{\circ} \mathrm{C}$ for $3 \mathrm{~d}$, the mycelium was overlaid with a solution of the starter unit AHBA in $10 \%$ DMSO diluted with water ( $\mathrm{pH}$ adjusted to $7 \cdot 2$ ) to give $1.5 \mathrm{ml}$ per plate. The agar in plates was allowed to dry for $45 \mathrm{~min}$ before use. After addition of the starter unit, incubation was continued for another $10 \mathrm{~d}$. The medium was then homogenized and extracted three times with an equal amount of ethyl acetate containing $1 \%$ acetic acid. The crude extract was pre-purified on a short $(5 \mathrm{~cm})$ silica gel column using $\mathrm{CHCl}_{3} / \mathrm{CH}_{3} \mathrm{OH}$ 4:1 (containing $1 \%$ acetic acid) as an eluant. All fractions containing product were combined and evaporated. The residue was rechromatographed on a $10 \mathrm{~cm}$ column using the same solvent system; the chromatographic purification removed most of the contaminants except AHBA. P8/1-OG was further purified by a second chromatography $\left(5 \% \mathrm{CH}_{3} \mathrm{OH}\right.$ in ethyl acetate, $1 \%$ acetic acid overall content) after application to the column in a small amount of $\mathrm{CHCl}_{3} / \mathrm{CH}_{3} \mathrm{OH} 4: 1$. Fractions containing pure product were combined and evaporated to give a yellowish residue that was identified as P8/1OG by: $R_{F}-0 \cdot 41\left(5 \% \mathrm{CH}_{3} \mathrm{OH}\right.$ in ethyl acetate, $1 \%$ acetic acid), $0.37\left(\mathrm{CHCl}_{3} / \mathrm{CH}_{3} \mathrm{OH} 4: 1,1 \%\right.$ acetic acid); HPLC (see below for conditions) $-t_{\mathrm{r}}=10 \cdot 28 \mathrm{~min} ;{ }^{1} \mathrm{H}-\mathrm{NMR}(400 \mathrm{MHz}$, $\mathrm{CD}_{3} \mathrm{OD}$ ) (refer to Ghisalba et al., 1981 for ${ }^{1} \mathrm{H}-\mathrm{NMR}$ data previously reported) $-6.24(\mathrm{t}, \mathrm{br}, 1 \mathrm{H}, J=1.60 \mathrm{~Hz}), 6 \cdot 16(\mathrm{t}, \mathrm{br}$, $1 \mathrm{H}, J=1 \cdot 46 \mathrm{~Hz}$ ), $6 \cdot 13(\mathrm{t}, 1 \mathrm{H}, J=2 \cdot 10 \mathrm{~Hz}), 6 \cdot 04(\mathrm{~s}, 1 \mathrm{H}), 4 \cdot 53$ (d, $1 \mathrm{H}, J=8.85 \mathrm{~Hz}$ ), 2.77 (qd, $1 \mathrm{H}, J=8.92,7.09 \mathrm{~Hz}$ ), 1.86 (s, $3 \mathrm{H}), 0.97(\mathrm{~d}, 3 \mathrm{H}, J=7 \cdot 02 \mathrm{~Hz}$ ) (br, broad; qd, quartet of doublets).

Quantitative HPLC analysis of production levels. 3-Hydroxybenzoic acid (10 mg per plate) was fed to both $A$. mediterranei HGF003 and A. mediterranei HZ149 as described above. Following incubation, culture media were extracted with ethyl acetate containing $1 \%$ acetic acid. The extracts were evaporated in vacuo and the residue was redissolved in $\mathrm{CH}_{3} \mathrm{OH}$ ( $2 \mathrm{ml}$ per plate extracted). The resulting solution was analysed by HPLC [Beckman ULTRASPHERE $\mathrm{C}_{18}, 5 \mu, 0 \cdot 46 \times 25 \mathrm{~cm}$; flow rate, $1.0 \mathrm{ml} \mathrm{min}{ }^{-1}$; injection volume, $20 \mu \mathrm{l}$; UV detection at 254 and $306 \mathrm{~nm}$; solvent gradient, $0-1 \mathrm{~min}$ : $100 \% \mathrm{~A}, 1-20$ $\min : 100 \% \mathrm{~A} \rightarrow 100 \% \mathrm{~B}, 20-25 \mathrm{~min}: 100 \% \mathrm{~B}$ (solvent A was $1 \%$ acetic acid in water and solvent $B$ was $1 \%$ acetic acid in $\left.\mathrm{CH}_{3} \mathrm{CN}\right)$ ]. The expected tetraketide eluted at $t_{\mathrm{r}}=13.06 \mathrm{~min}$.

\section{RESULTS}

\section{Cloning the rifamycin PKS gene cluster}

Although an earlier report (August et al., 1998) has described the cloning and sequence analysis of the entire rifamycin gene cluster, most of the primary genomic fragments isolated in that study were relatively small $(<20 \mathrm{~kb})$. Because this presented a problem for the genetic strategy described below, the rifamycin PKS genes were recloned as cosmid-sized inserts. When the $7 \cdot 5 \mathrm{~kb}$ Pst I fragment from pIY5 was labelled with DIG and used to probe 2000 colonies from the library, 42 hybridizing clones were detected. Of these cosmid clones, $\cos 2$, $\cos 6$ and $\cos 42$ (Fig. 1) were shown by comparing their restriction sites with published sequences of the rifamycin PKS genes (Tang et al., 1998) to cover the entire rifamycin PKS gene cluster.

\section{Construction of a deletion host}

DNA fragments $\mathrm{A}, \mathrm{B}$ and $\mathrm{C}$ were subcloned from cosmids $\cos 2, \cos 42$ and cos6, respectively (Fig. 2). Fragment A starts at nt 651 and continues to nt 1438 (where nucleotides are numbered from the first base of the rifamycin PKS genes). Fragment B extends from nt 28464 to nt 29417, and includes the end of module 6 as well as the start of module 7 . Fragment $\mathrm{C}$ contains the end of module 10 starting from nt 47393 and continues to nt 50159; it includes the $3^{\prime}$ end of the rifF amide 


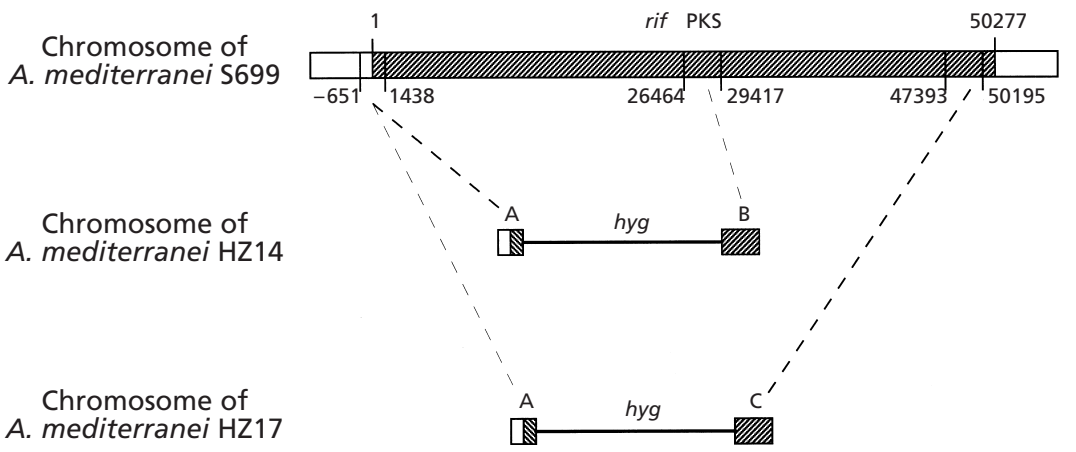

Fig. 2. Construction of the deletion hosts $\mathrm{HZ14}$ and HZ17. The relative positions of fragments $A, B$ and $C$ within the rifamycin PKS gene cluster (hatched) are shown. Position 1 refers to the first $G$ of the start codon (GTG). In A. mediterranei $\mathrm{HZ14}$ the first six modules were replaced by a hyg cassette, whereas in A. mediterranei HZ17 all 10 modules were replaced with the same hyg cassette.

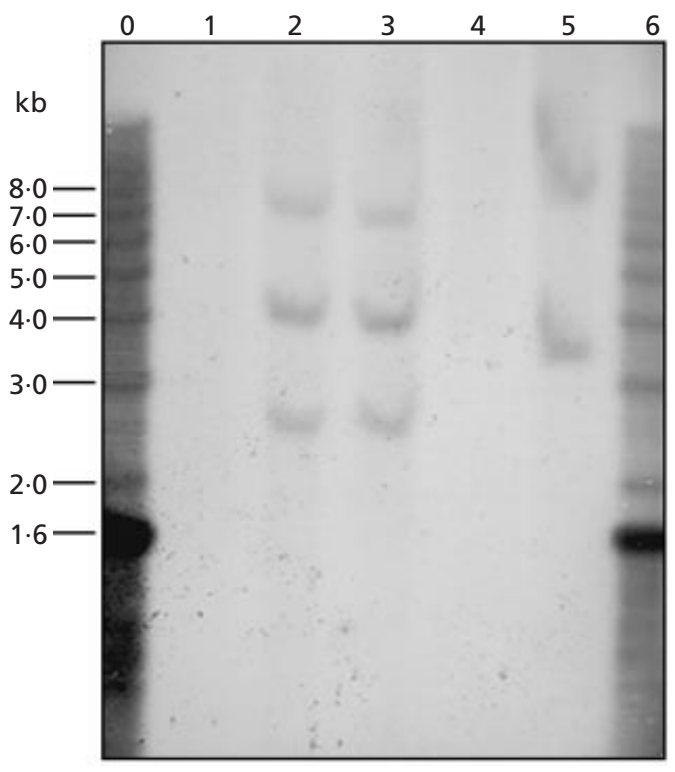

Fig. 3. Southern hybridization of genomic DNA from the deletion hosts $\mathrm{HZ14}$ and $\mathrm{HZ17}$. The hyg gene fragment was used as the probe. Lanes: 0 and 6, $1 \mathrm{~kb}$ ladder (Gibco-BRL); 1, genomic DNA from $A$. mediterranei $\$ 699$ digested with $E c o R I+X m n l ; 2$ and 3, genomic DNA from two independent isolates of $A$. mediterranei $\mathrm{HZ17}$ digested with $E \mathrm{CoRI}+X m n l ; 4$, genomic DNA from $A$. mediterranei $\$ 699$ digested with EcoRI+HindIII; 5, genomic DNA from A. mediterranei HZ14 digested with EcoRI + HindIII. The expected bands from $\mathrm{HZ14}$ had sizes of 3.2 and $7.8 \mathrm{~kb}$, whereas the expected bands from HZ17 had sizes of 2.6 and $4.1 \mathrm{~kb}$. An additional band from $\mathrm{HZ17}$, at $6.7 \mathrm{~kb}$, is presumably caused by partial digestion of the genomic DNA.

synthase gene. Two suicide plasmids were constructed in pSET152 (NheI-EcoRI) with the hygromycin-resistance gene (byg) (Malpartida et al., 1983) flanked by either fragments $\mathrm{A}$ and $\mathrm{B}$ or $\mathrm{A}$ and $\mathrm{C}$ in their natural orientations. These constructs, designated pHU130 and pHU131, respectively, were introduced into A. mediterranei $\mathrm{S} 699$ by electroporation. Approximately 10 $h_{y g^{+}} a_{p r^{+}}$colonies were obtained from each transformation, presumably as a result of a single crossover in one of the homologous regions. One such transformant was randomly selected in each case, and propagated through two rounds of subsequent growth on non-selective YMG agar. More than 200 single colonies were tested for resistance to apramycin and hygromycin on selective YMG plates. Two derivatives of pHU130::S699 and three derivatives of pHU131::S699 had lost their resistance to apramycin but were still resistant to hygromycin. Southern analysis of XmnI + EcoRI-digested genomic DNA from two of the three pHU131::S699 derivatives, and of HindIII + EcoRI-digested genomic DNA from one of the two pHU130::S699 derivatives, using the byg gene as a probe, confirmed that all three colonies were derived from double-crossover events, as expected (Fig. 3). Randomly chosen derivatives of pHU130::S699 and pHU131::S699 were designated A. mediterranei strains HZ14 and HZ17, respectively (see Fig. 2).

Strains HZ14 and HZ17 were grown in YMG liquid medium for $10 \mathrm{~d}$ at $30{ }^{\circ} \mathrm{C}$ with constant shaking at 250 r.p.m. Supernatants from the cultures were extracted three times with ethyl acetate containing $1 \%$ acetic acid. The organic phases were evaporated and the crude extracts were resuspended in ethyl acetate. TLC analysis of these extracts using authentic AHBA as a reference revealed that both cultures had produced significant amounts of AHBA (the starter unit for rifamycin synthesis). This was confirmed by co-synthesis experiments using these strains as secretors and A. mediterranei HGF003 (which contains an inactive AHBA synthase gene) as a convertor strain. The mixed fermentations produced rifamycin $\mathrm{B}$, detected by HPLCUV analysis (data not shown). Together, these results showed that the biosynthesis of AHBA was not affected by the deletions in A. mediterranei HZ14 and HZ17.

\section{Expression of a truncated form of the rifamycin PKS in a deletion host}

The XmnI (position -651)-RcaI (position 14757) fragment from cos2 was end-filled using T4 DNA polymerase and cloned into the SmaI site of pUC119 to give $\mathrm{pHU} 42$. The $\mathrm{Xba \textrm {I } - E c o R I}$ fragment from $\mathrm{pHU} 42$ was then ligated with NheI + EcoRI-digested pSET152 to give the plasmid pHU149. Plasmid pHU149 was introduced into the deletion host A. mediterranei strain HZ17 by electroporation. Two apramycin-resistant 

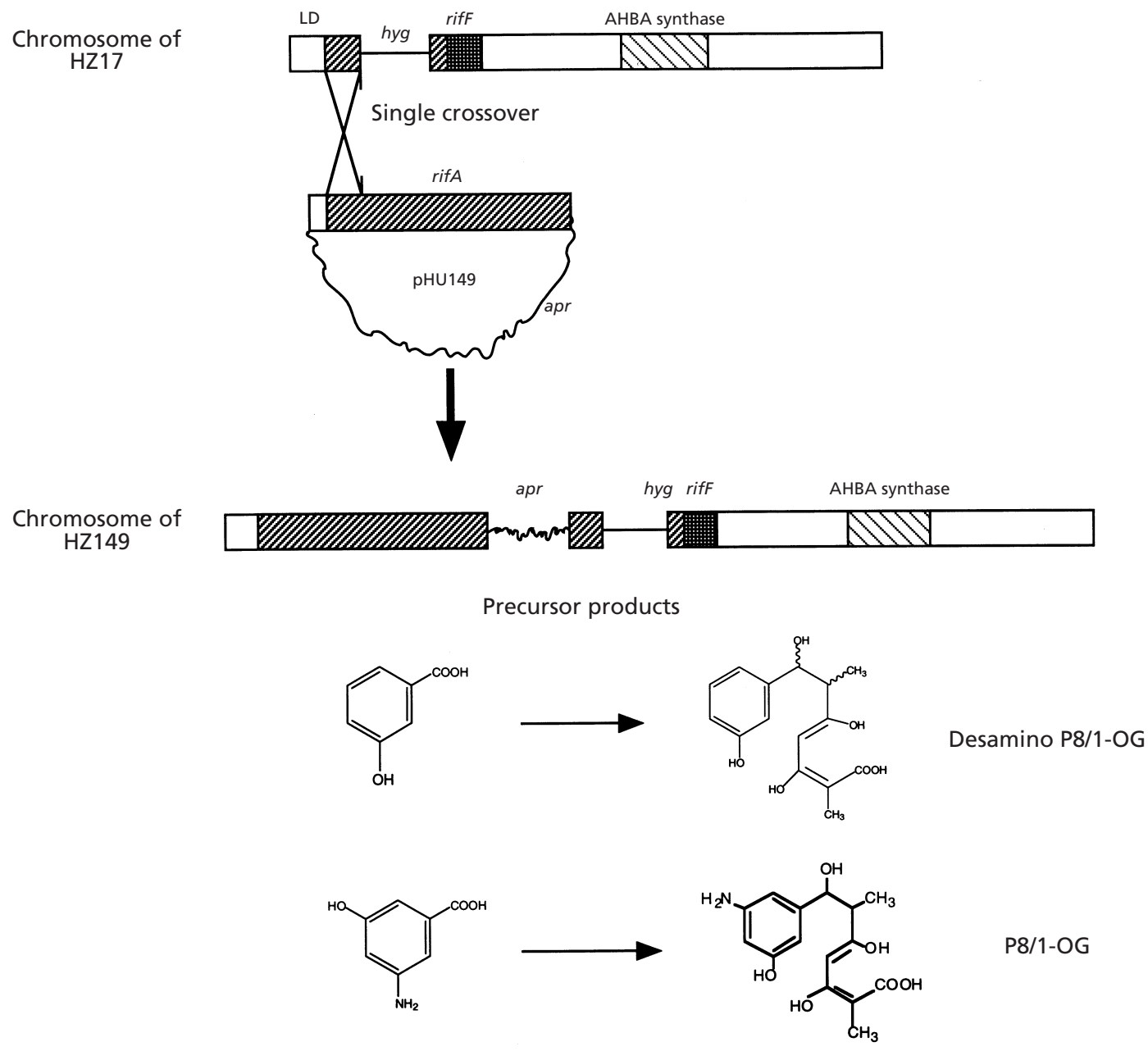

Fig. 4. Construction and analysis of HZ149. The strategy for constructing strain $\mathrm{HZ149}$ is shown. The Xmnl (position -651)-Rcal (position 14757) fragment from cos2 (Fig. 1) contains rifA, which encodes AHBA-CoA ligase and the first three modules of the rifamycin PKS. Integration of this fragment by a single crossover into the genome of $\mathrm{HZ17}$ yielded HZ149, which was unable to produce either AHBA or P8/1-OG by itself, but produced P8/1-OG or its desamino analogue when supplemented with AHBA or 3-hydroxybenzoic acid, respectively. LD, Loading domain; apr, apramycin-resistance gene; hyg, hygromycin-resistance gene.

transformants, HZ149-1 and HZ149-3, were obtained (Fig. 4). Extraction with ethyl acetate containing $1 \%$ acetic acid and TLC comparison with a comparable extract from the deletion host HZ17 revealed no major metabolite in the fermentation medium. Moreover, no AHBA was produced by either transformant. Analysis by reverse-phase HPLC yielded the same result, leading us to suspect that HZ149-1 or HZ149-3 might be capable of producing tetraketides, but that tetraketide production was down-regulated due to attenuated biosynthesis of AHBA. Therefore, A. mediterranei strains HZ149-3, HZ17 and HGF003 were subjected to more detailed metabolite analysis.

\section{Metabolite analysis of HZ149}

A. mediterranei HZ149-3 was grown on YMG agar for $10 \mathrm{~d}$ in the presence of exogenous AHBA $(10 \mathrm{mg}$ per plate). The crude product obtained by extraction with ethyl acetate containing $1 \%$ acetic acid was analysed for polyketide metabolites by TLC and HPLC. A major new product was isolated and identified as P8/1-OG (Fig. 4) from NMR spectroscopic data. Based on the amount of P8/1-OG recovered, the titre was calculated to be $\approx 30 \mathrm{mg} \mathrm{l}^{-1}$.

Earlier studies had shown that A. mediterranei HGF003 is capable of producing polyketides in high yields when fed a variety of exogenous starter units (Hunziker et al., 1998). However, whereas AHBA is converted into rifamycin B by this strain, alternative starter units such as 3-hydroxybenzoic acid and 3,5-dihydroxybenzoic acid are processed only into tetraketide analogues of P8/1-OG. Therefore, the production levels of A. mediterranei strains HZ149-3 and HGF003 were compared by adding 3-hydroxybenzoic acid to growing cultures of both strains. Following incubation, culture extracts were analysed by HPLC for tetraketide (desamino P8/1- 
OG) levels. Desamino P8/1-OG production by HZ149 was approximately $35 \%$ relative to HGF003. Purification of desamino P8/1-OG from the HZ149-3 extract gave $7.7 \mathrm{mg}$ pure compound, which corresponded to a titre of approximately $50 \mathrm{mg} \mathrm{l}^{-1}$. HGF003 has been shown previously to produce desamino P8/1-OG at $135 \mathrm{mg} \mathrm{l}^{-1}$ (Hunziker et al., 1998).

\section{DISCUSSION}

We describe the construction of two potentially useful hosts for manipulating rifamycin biosynthesis, and use one of them (the 10-module deletion host) to successfully express the rifA gene. We expect that the host-vector system described here will be valuable in further studies on the rifamycin PKS. There are several advantages to manipulating the rifamycin PKS in its original host, $A$. mediterranei S699. First, A. mediterranei produces a large amount of rifamycin $\left(>1 \mathrm{~g} \mathrm{l}^{-1}\right)$ in stirred-tank fermentations. Since PKS manipulation often leads to reduced production of reporter polyketides, this enhanced productivity can facilitate isolating 'unnatural' natural products. Second, the rifamycin biosynthetic pathway includes several post-PKS tailoring steps, many of which are poorly characterized at the genetic and enzymological level. By using A. mediterranei one ensures that all downstream enzymes are available and that at least some modified cores can be converted into rifamycin $\mathrm{B}$ analogues. Third, the A. mediterranei derivatives described here offer relatively easy access to any combinatorial mutants in the 10 modules through the use of either the full 10-module deletion host (HZ17) or the six-module deletion host (HZ14). Finally, as other important biosynthetic gene clusters from Amycolatopsis species are cloned, the deletion hosts could also be useful for facile genetic engineering of heterologous biosynthetic pathways. For example, HZ14 and HZ17 could also be used to study genes from the recently described chloroeremomycin biosynthetic gene cluster from A. orientalis (Van Wageningen et al., 1998).

Although A. mediterranei is taxonomically related to Streptomyces, methods for gene cloning in A. mediterranei are relatively underdeveloped. In particular, a suitable low-copy plasmid capable of holding large inserts is not yet available. Moreover, the absence of endogenous promoters suitable for polyketide gene expression is a disadvantage. Because our host-vector strategy uses a suicide vector to insert the desired gene(s) into the chromosome of $A$. mediterranei through a selectable single crossover, the expressed genes can use the same promoter that is presumably responsible for transcription of the rifamycin PKS genes. Although we were able to generate HZ149 in a single-step process, we were unable to deliver very large fragments (approx. $50 \mathrm{~kb}$, carrying the first six modules of the rifamycin PKS gene cluster) into the chromosomes of the deletion hosts (data not shown). This may be due to either poor transfer of extremely large fragments into A. mediterranei, or the greater likelihood of their destruction by endogenous nucleases. As improved plasmids and pro- moters are developed for A. mediterranei, we expect that the utility of our deletion hosts will increase further.

Finally, it is intriguing that production of P8/1-OG in HZ149 depends on the addition of exogenous AHBA. Since both wild-type A. mediterranei and its deletion derivatives can produce large amounts of AHBA, our results may suggest that one or more genes downstream of the rifamycin PKS operon are required for AHBA biosynthesis, and that integration of a suicide vector disrupts their transcription. Further experiments may help resolve this interesting conundrum.

\section{ACKNOWLEDGEMENTS}

This paper is dedicated to Professor Sir David A. Hopwood, FRS, for his extraordinary vision and pioneering contributions in the field of actinomycete biology and chemistry. We thank Dr Tin-Wein Yu (University of Washington) and Dr Li Tang (University of Wisconsin) for helpful advice on transforming A. mediterranei. This work was supported by a grant from the National Institutes of Health (AI 38947) to C.R.H. and C.K.

\section{REFERENCES}

Arora, S. K. (1985). Correlation of structure and activity in ansamycins: structure, conformation, and interactions of antibiotic rifamycin S. J Med Chem 28, 1099-1102.

Arora, S. K. \& Main, P. (1984). Correlation of structure and activity in ansamycin: molecular structure of cyclized rifamycin SV. J Antibiot 37, 178-181.

August, P. R., Tang, L., Yoon, Y. J. \& 9 other authors (1998). Biosynthesis of the ansamycin antibiotic rifamycin. Deductions from the molecular analysis of the rif biosynthetic gene cluster of Amycolatopsis mediterranei S699. Chem Biol 5, 69-79.

Bacchi, A., Pelizzi, G., Nebuloni, M. \& Ferrari, P. (1998). Comprehensive study on structure-activity relationships of rifamycins: discussion of molecular and crystal structure and spectroscopic and thermochemical properties of rifamycin O. J Med Chem 41, 2319-2332.

Barna, J. C. J. \& Williams, D. H. (1984). The structure and mode of action of glycopeptide antibiotics of the vancomycin group. Annu Rev Microbiol 38, 339-357.

Bartolucci, C., Cellai, L., Marzano, M. \& 8 other authors (1995). Structure-activity relationships in open ansa-chain rifamycin $\mathrm{S}$ derivatives as inhibitors of HIV-1 reverse transcriptase. Farmaco 50, 587-593.

Bedford, D., Jacobsen, J. R., Luo, G., Cane, D. E. \& Khosla, C. (1996). A functional chimeric modular polyketide synthase generated via domain replacement. Chem Biol 3, 827-831.

Cane, D. E., Walsh, C. T. \& Khosla, C. (1998). Harnessing the biosynthetic code. Combinations, permutations, mutations. Science 282, 63-68.

Cole, S. T. (1996). Rifamycin resistance in mycobacteria. Res Microbiol 147, 48-52.

Donadio, S., McAlpine, J. B., Sheldon, P. J., Jackson, M. \& Katz, L. (1993). An erythromycin analog produced by reprogramming of polyketide synthesis. Proc Natl Acad Sci USA 90, 7119-7123.

Flett, F., Mersinias, V. \& Smith, C. P. (1997). High efficiency intergeneric conjugal transfer of plasmid DNA from Escherichia coli to methyl DNA-restricting streptomycetes. FEMS Microbiol Lett 155, 233-239. 
Ghisalba, O. \& Nuesch, J. (1981). A genetic approach to the biosynthesis of the rifamycin-chromophore in Nocardia mediterranei. IV. Identification of 3-amino-5-hydroxybenzoic acid as a direct precursor of the seven-carbon amino starter-unit. J Antibiot 34, 64-71.

Ghisalba, O., Fuhrer, H., Richter, W. J. \& Moss, S. (1981). A genetic approach to the biosynthesis of the rifamycin-chromophore in Nocardia mediterranei. III. Isolation and identification of an early aromatic ansamycin-precursor containing the seven-carbon amino starter-unit and three initial acetate/propionate-units of the ansa chain. J Antibiot 34, 59-63.

Hatano, K., Akiyama, S., Asai, M. \& Rickards, R. W. (1982). Biosynthetic origin of aminobenzenoid nucleus $\left(\mathrm{C}_{7} \mathrm{~N}\right.$-unit $)$ of ansamitocin, a group of novel maytansinoid antibiotics. $J$ Antibiot 35, 1415-1417.

Hunziker, D., Yu, T. W., Hutchinson, C. R., Floss, H. G. \& Khosla, C. (1998). Primer unit specificity in rifamycin biosynthesis principally resides in the later stages of the biosynthetic pathway. J Am Chem Soc 120, 1092-1093.

Kao, C. M., Katz, L. \& Khosla, C. (1994). Engineered biosynthesis of a complete macrolactone in a heterologous host. Science $\mathbf{2 6 5}$, 509-512.

Kao, C. M., Pieper, R., Cane, D. E. \& Khosla, C. (1996). Evidence for two catalytically independent clusters of active sites in a functional modular polyketide synthase. Biochemistry 135, 12363-12368.

Kim, C. G., Yu, T. W., Fryhle, C. B., Handa, S. \& Floss, H. G. (1998). 3-Amino-5-hydroxybenzoic acid synthase, the terminal enzyme in the formation of the precursor of $\mathrm{mC}_{7} \mathrm{~N}$ units in rifamycin and related antibiotics. J Biol Chem 273, 6030-6040.

Lal, R., Lal, S. \& Grund, E. (1991). Construction of a hybrid plasmid capable of replication in Amycolatopsis mediterranei. Appl Environ Microbiol 57, 665-671.

Lal, R., Khanna, R., Dhingra, N., Khanna, M. \& Lal, S. (1998). Development of an improved cloning vector and transformation system in Amycolatopsis mediterranei (Nocardia mediterranei). J Antibiot 51, 161-169.

Lowder, J. F. \& Johnson, R. S. (1987). The generation of the rifamycin binding site in the beta subunit of E. coli RNA polymerase through subunit interactions. Biochem Biophys Res Commun 147, 1129-1136.

McDaniel, R., Ebert-Khosla, S., Hopwood, D. A. \& Khosla, C. (1993). Engineered biosynthesis of novel polyketides. Science 262, 1546-1550.

Madon, J. \& Hutter, R. (1991). Transformation system for Amycolatopsis (Nocardia) mediterranei: direct transformation of mycelium with plasmid DNA. J Bacteriol 173, 6325-6331.

Malpartida, F., Zalacain, M., Jimenen, A. \& Davies, J. (1983). Molecular cloning and expression in Streptomyces lividans of a hygromycin B phosphotransferase gene from Streptomyces hygroscopicus. Biochem Biophys Res Commun 117, 6-12.

Marsden, A. F., Wilkinson, B., Cortes, J., Dunster, N. J., Staunton, J. \& Leadlay, P. F. (1998). Engineering broader specificity into an antibiotic-producing polyketide synthase. Science 279, 199-202.

Matsushima, P., McHenney, M. A. \& Baltz, R. H. (1987). Efficient transformation of Amycolatopsis orientalis (Nocardia orientalis) protoplasts by Streptomyces plasmids. J Bacteriol 169, 2298-2300.

Moretti, P., Hintermann, G. \& Huttler, R. (1985). Isolation and characterization of an extrachromosomal element from Nocardia mediterranei. Plasmid 14, 126-133.
Nadkarni, S. R., Patel, M. V., Chatterjee, S., Vijayakumar, E. K. S., Desikan, K. R., Blumbach, J. \& Ganguli, B. N. (1994). Balhimycin, a new glycopeptide antibiotics produced by Amycolatopsis sp. Y86,21022 . Taxonomy, production, isolation and biological activity. J Antibiot 47, 334-341.

Pelzer, S., Reichert, W., Huppert, M., Heckmann, D. \& Wohlleben, W. (1997). Cloning and analysis of a peptide synthetase gene of the balhimycin producer Amycolatopsis mediterranei DSM5908 and development of a gene disruption/replacement system. $J$ Biotechnol 56, 115-128.

Riva, S., Fietta, A. \& Silvestri, L. G. (1972). Mechanism of action of a rifamycin derivative (AF-013) which is active on the nucleic acid polymerases insensitive to rifampicin. Biochem Biophys Res Commun 49, 1263-1271.

Rowe, C. J., Cortes, J., Gaisser, S., Staunton, J. \& Leadlay, P. F. (1998). Construction of new vectors for high-level expression in actinomycetes. Gene 216, 215-223.

Ruan, X., Pereda, A., Stassi, D. L. \& 8 other authors (1997). Acyltransferase domain substitutions in erythromycin polyketide synthase yield novel erythromycin derivatives. J Bacteriol 179, 6416-6425.

Sambrook, J., Fritsch, E. F. \& Maniatis, T. (1989). Molecular Cloning: a Laboratory Manual, 2nd edn. Cold Spring Harbor, NY: Cold Spring Harbor Laboratory.

Schupp, T. \& Diver, M. (1986). Protoplast preparation and regeneration in Nocardia mediterranei. FEMS Microbiol Lett 36, 159-165.

Schupp, T., Toupet, C., Engel, N. \& Goff, S. (1998). Cloning and sequence analysis of the putative rifamycin polyketide synthase gene cluster from Amycolatopsis mediterranei. FEMS Microbiol Lett 157, 201-207.

Sensi, P., Margalith, P. \& Timbal, M. T. (1959). Rifamycin, a new antibiotic. Preliminary report. Farm Ed Sci 14, 146-147.

Tang, L., Yoon, Y. J., Choi, C. Y. \& Hutchinson, C. R. (1998). Characterization of the enzymatic domains in the modular polyketide synthase involved in rifamycin B biosynthesis by Amycolatopsis mediterranei. Gene 216, 255-265.

Ueno, M., Iijima, M., Masuda, T., Kinoshita, N., linuma, H., Naganawa, H., Hamada, M., Ishizuka, M. \& Takeuchi, T. (1992). Dethymicin, a novel immunosuppressant isolated from an Amycolatopsis. Fermentation, isolation, physico-chemical properties and biological activities. J Antibiot 12, 1819-1826.

Van Wageningen, A. M., Kirkpatrick, P. N., Williams, D. H., Harris, B. R., Kershaw, J. K., Lennard, N. J., Jones, M., Jones, S. J. \& Solenberg, P. J. (1998). Sequencing and analysis of genes involved in the biosynthesis of a vancomycin group antibiotic. Chem Biol 5, 133-162.

Vrijbloed, J. W., Madon, J. \& Dijkhuizen, L. (1994). A plasmid from the methylotrophic actinomycete Amycolatopsis methanolica capable of site-specific integration. J Bacteriol 176, 7087-7090.

Yu, T.-W., Shen, Y., Doi-Katayama, Y., Tang, L., Park, C., Moore, B. S., Hutchinson, C. R. \& Floss, H. G. (1999). Direct evidence that the rifamycin polyketide synthase assembles polyketide chains processively. Proc Natl Acad Sci USA (in press).

Received 23 February 1999; revised 18 May 1999; accepted 25 May 1999. 